\title{
Prevalencia del hipogonadismo entre hombres infectados con VIH y su relación con los niveles de CD4: Evidencia desde Quito, Ecuador
}

\author{
Prevalence of hypogonadism among men infected with HIV and its \\ association with CD4 levels: Evidence from Quito, Ecuador
}

Prevalência de hipogonadismo entre homens infectados pelo HIV e sua relação com os níveis de CD4: Evidências de Quito, Equador

\author{
Fernando Rafael Rodríguez Ibarra (iD ${ }^{a}$, Ixsoon Alberto Castillo ${ }^{b}$, \\ Patricia Lisseth Borrero Párraga (iD ${ }^{a}$, Tatiana Carolina Marcillo Pin (iD) ${ }^{a}$
}

\begin{abstract}
a Servicio de Medicina Interna, Clínica Guayaquil, GuayaquilEcuador; ' ${ }^{\circ}$ Unidad Integral de VIH, Hospital de Especialidades Eugenio Espejo, Quito-Ecuador.
\end{abstract}

\section{Correspondencia a:}

Fernando Rafael Rodríguez Ibarra, dr.rodriguez@hotmail.es

Recibido: 15 de octubre, 2020 Aceptado: 11 de diciembre, 2020 Publicado: 4 de enero, 2021

\section{ARTÍCULO ORIGINAL}

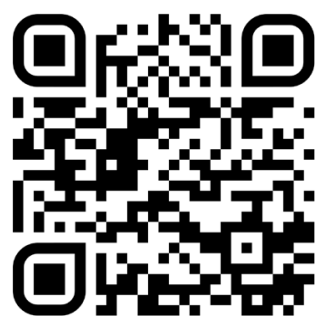

Escanea en tu dispositivo móvil o revisa este artículo en: https:// revistaclinicaguayaquil.org

\section{RESUMEN}

Introducción: Para el 2017 alrededor de 36.9 millones de personas eran portadoras del virus de la inmunodeficiencia humana $(\mathrm{VIH})$, de las cuales, solo 21.7 millones recibían terapia antirretrovírica. Varias investigaciones exploratorias evidencian una alta tasa de incidencia de hipogonadismo en pacientes con $\mathrm{VIH}$, observando cierta relación entre los niveles de CD4 y testosterona. Este trabajo analiza el impacto de los niveles de células CD4 sobre la posibilidad de ser diagnosticado con hipogonadismo en hombres infectados con $\mathrm{VIH}$.

Materiales y Métodos: Se estimó un modelo Logit en el que la variable dependiente toma valores de 1 cuando el sujeto de muestra es diagnosticado con hipogonadismo y 0 para el caso contrario. Adicionalmente se incluyeron al modelo variables como la edad y los niveles de hormonas LH y FSH.

Resultados: Se incluyeron 114 hombres con $\mathrm{VIH}$, con edad media de 35 años, de los cuales el 6\% padecía de hipogonadismo. Además, presentaron una media de niveles de testosterona de $274.45 \mathrm{ng} / \mathrm{dl}$, de recuento de células CD4 de $376.04 \mathrm{c} / \mathrm{mm}^{3}$, y de LH y FSH de 5.89 $\mathrm{mIU} / \mathrm{ml}$ y $5.68 \mathrm{mlU} / \mathrm{ml}$, respectivamente.

Conclusiones: Se evidenció la ausencia de un impacto estadísticamente significativo de los niveles de células CD4 sobre la posibilidad de contraer hipogonadismo en los hombres con VIH. Además, al evaluar si la edad de los participantes tiene algún efecto sobre la presencia de la enfermedad, se halló que este factor tampoco tiene relevancia.

Palabras clave: Virus de la inmunodeficiencia humana; síndrome de inmunodeficiencia adquirida; hipogonadismo; antígenos CD4; SIDA

\footnotetext{
ABSTRACT

Introduction: Human immunodeficiency virus (HIV) infection is known worldwide, by 2017 around 36.9 million people were carriers, of which only 21.7 million were receiving antiretroviral therapy. Several exploratory investigations have shown a high incidence of hypogonadism in patients with HIV, observing a certain relationship between CD4 and testosterone levels. This work analyzes the impact of CD4 cell levels on the possibility of being diagnosed with hypogonadism in HIV-infected men.

Materials and Methods: A Logit model was estimated in which the dependent variable takes values of 1 when the sample subject is diagnosed with hypogonadism and 0 for the opposite case.
} 
Additionally, variables proposed by previous studies were included in the model, such as age and levels of hormones LH and FSH .

Results: A hundred and fourteen men with HIV were included, with a mean age of 35 years, of which $6 \%$ suffered from hypogonadism. The average level of testosterone was $274.45 \mathrm{ng} / \mathrm{dl}$, a mean CD4 cell count of $376.04 \mathrm{c} / \mathrm{mm}^{3}$, and a mean LH and FSH of $5.89 \mathrm{mlU} / \mathrm{ml}$ and $5.68 \mathrm{mlU} /$ $\mathrm{ml}$, respectively.

Conclusions: The analysis showed the absence of a statistically significant impact of CD4 cell levels on the possibility of contracting hypogonadism in men with HIV. Furthermore, when evaluating whether the age of the participants had any effect on the presence of the disease, it was found that this factor is also irrelevant.

Key words: Human immunodeficiency virus; acquired immunodeficiency syndrome; hypogonadism; CD4 antigens; AIDS

\section{RESUMO}

Introdução: Em 2017, cerca de 36,9 milhões de pessoas eram portadoras do vírus da imunodeficiência humana(HIV), das quais apenas 21,7 milhões recebiam terapia antirretroviral. Diversas investigações exploratórias mostram uma alta taxa de incidência de hipogonadismo em pacientes com HIV, observando certa relação entre os níveis de CD4 e testosterona. Este trabalho analisa o impacto dos níveis de células CD4 na possibilidade de diagnóstico de hipogonadismo em homens infectados pelo HIV. Materiais e Métodos: Foi estimado um modelo Logit no qual a variável dependente assume valores de 1 quando o sujeito da amostra é diagnosticado com hipogonadismo e 0 para o caso oposto. Além disso, variáveis como idade e níveis dos hormônios LH e FSH foram incluídas no modelo.

Resultados: Foram incluídos 114 homens com HIV, com média de idade de 35 anos, dos quais $6 \%$ sofriam de hipogonadismo. Além disso, eles tinham um nível médio de testosterona de $274,45 \mathrm{ng} / \mathrm{dl}$, uma contagem de células CD4 de $376,04 \mathrm{c} / \mathrm{mm}^{3}$ e LH e FSH de 5,89 $\mathrm{mlU} / \mathrm{ml}$ e 5,68 $\mathrm{mIU} / \mathrm{ml}$, respectivamente.

Conclusões: Evidenciou-se a ausência de impacto estatisticamente significativo dos níveis de células CD4 sobre a possibilidade de contrair hipogonadismo em homens com HIV. Além disso, ao avaliar se a idade dos participantes influencia na presença da doença, constatou-se que esse fator também é irrelevante.

Palavras-chave: Vírus da imunodeficiência humana; síndrome da imunodeficiência adquirida; hipogonadismo; antígenos CD4; AIDS

\section{INTRODUCCIÓN}

En el 2017 alrededor de 36.9 millones de personas eran portadores del virus de inmunodeficiencia humana $(\mathrm{VIH})$ en el mundo, de las cuales, solo
21.7 millones recibían la terapia antirretrovírica (1). A su vez, el 70\% de la población contagiada que no recibe tratamiento, vive en países en vías de desarrollo (1). Aunque la introducción del tratamiento antirretroviral mermó la tasa de mortalidad en los contagiados, esta trajo nuevas afecciones relacionadas al sistema endócrino, provocando un desconcierto entre los académicos por su impacto en la presentación e interpretación de los resultados bioquímicos (2).

Previa aparición de las primeras terapias antirretrovirales en 1985, varias investigaciones exploratorias evidenciaron una alta tasa de incidencia de hipogonadismo en pacientes con $\mathrm{VIH}$, observando cierta relación entre los niveles de CD4 y testosterona (3). El patrón observado mostraba una baja cantidad de CD4 en las primeras etapas del $\mathrm{VIH}$, reflejando un incremento de los niveles de testosterona, mientas que, en las últimas etapas de la afección, se producía una disminución de esta (2). Durante dicho proceso, el $50 \%$ de los contagiados con VIH sufrían de hipogonadismo (2).

En la era post tratamiento, la prevalencia de hipogonadismo en los pacientes con VIH de etapa terminal se redujo a tasas que oscilaban entre el $29 \%$ y $50 \%$, revelando una fuerte persistencia de la afección entre los contagiados, incluso desde las primeras etapas del $\mathrm{VIH}$ (4-6). Al mismo tiempo, varios trabajos exponían la ausencia de diferencias significativas en los niveles de testosterona, entre quienes reciben y no reciben el tratamiento, desvinculando el uso del mismo con la presencia del hipogonadismo $(7,8)$. Por ello, otras investigaciones intentaron vincular el hipogonadismo con factores no asociados al $\mathrm{VIH}$, como el abuso de drogas ilícitas, esteroides y el alcohol (4).

La motivación de los científicos dedicados a investigar el hipogonadismo en los pacientes con VIH, radica en la búsqueda de alternativas médicas que mejoren la calidad de vida de los pacientes pese a su diagnóstico (2). Es decir, en vista de que el tratamiento antirretroviral provee longanimidad a sus pacientes; el cómo el hipogonadismo afecta su vida sexual, resulta de interés para ellos.

A pesar de que las indagaciones plantean una relación entre CD4, la cantidad de testosterona e incluso la duración del tratamiento, debido a las anormalidades metabólicas y endocrinas provocadas por el virus, no existe mayor información que permita ser concluyente acerca de los antiretrovirales como origen 
del hipogonadismo (4). Dadas las distintas concepciones causales de dicha afección, el siguiente documento busca corroborar el impacto de los niveles de CD4 sobre la incidencia del hipogonadismo en hombres infectados con el VIH, por medio de una evaluación del aumento o disminución de células CD4 y cómo este factor repercute en el nivel de testosterona, siendo este último, una variable determinante para el diagnóstico de la enfermedad.

\section{MATERIALES Y MÉTODOS}

Este estudio fue ejecutado en el hospital Eugenio Espejo de la ciudad de Quito, Ecuador, con previo consentimiento informado de las autoridades de la institución. Para motivar a los pacientes varones infectados de $\mathrm{VIH}$ a participar en el estudio, se diseñó un programa de incentivos a través de campañas informativas sobre los beneficios de la investigación científica en el bienestar sexual de los afectados. Se obtuvo un total de 114 pacientes varones inscritos con edades entre 18 y 75 años, los cuales fueron sometidos a una evaluación clínica que comprendió pruebas médicas y revisión documental del historial clínico.

Para diagnosticar el hipogonadismo se utilizó un examen de sangre que fue testeado a través del operador Immulite 2000 que, si bien no es el gold estándar, es el único método que disponía el hospital para detectar los niveles de testosterona. Luego, tomando las recomendaciones de la guía práctica de la Sociedad Endócrina (9), se calificó como pacientes hipogonadales a aquellos que tuvieron niveles de testosterona menores a 300 $\mathrm{ng} / \mathrm{dl}$, grupo que representa el $6 \%$ de la muestra $y$, como pacientes no hipogonadales a aquellos con niveles de testosterona mayores o iguales a $300 \mathrm{ng} / \mathrm{dl}$.

Por otra parte, el recuento de células CD4 fue estimado a partir de la revisión del historial clínico de los participantes, para luego ser categorizados en 4 rangos distintos. El primer rango incluye aquellos participantes que mantienen niveles de CD4 inferiores o iguales a $200 \mathrm{c} / \mathrm{mm}^{3}$, grupo que según los Centros para el Control de Enfermedades de Estados Unidos (CDC) y la Organización Mundial de la Salud (OMS) son clasificados como SIDA, y por último, el cuarto grupo engloba aquellos pacientes con recuento de CD4 superiores a $500 \mathrm{c} / \mathrm{mm}^{3}$, que se interpretan como recuentos normales (10).

Adicionalmente y siguiendo los trabajos de Ranabir et. al. (7), Laudat et. al. (11), Wong et. al.
(2) se calcularon los niveles de hormona folículo estimulante (FSH) y hormona luteinizante (LH) en cada paciente mediante el método de quimioluminiscencia, con el objetivo de ingresarlo al análisis como factores incidentes del hipogonadismo. Además, se tomó como referencia el trabajo de Bajaj et al. (12) para incluir la edad como una variable de control que influye de forma transitoria en la producción de testosterona, sin embargo, este trabajo incluye en el modelo la edad en términos cuadráticos debido al supuesto comportamiento no lineal de la variable, esto es, a medida que la edad del paciente aumenta, la producción normal de testosterona, disminuye. Ciertamente, este trabajo tiene como objetivo corroborar el impacto de las células CD4 sobre la posibilidad de contraer hipogonadismo en pacientes varones con $\mathrm{VIH}$, es decir, si la disminución o aumento de niveles de células CD4 repercute en la prevalencia de la enfermedad. Sin embargo, se consideró apropiado incluir variables propuestas en trabajos precedentes para evitar el sesgo de sobreestimación o subestimación, por ello, se designaron los niveles de CD4, LH, y FSH junto con la edad del paciente como variable independiente $\mathrm{y}$ el hipogonadismo como variable dependiente.

Por lo tanto, dada la naturaleza de la variable dependiente, que tiene valores de 1 cuando el paciente padece de hipogonadismo, y 0 cuando no mantiene esta afección, se utilizó un modelo de elección discreta en el que la variable dependiente responde a la ausencia o presencia de hipogonadismo en los sujetos de prueba. De igual manera, se evaluó el efecto de las células CD4 a partir de los datos categorizados, con el objetivo de estimar los cambios discretos respecto de una categoría base, que permite una interpretación más apropiada al fenómeno de estudio. Específicamente, se estimó un modelo Logit que utiliza como función de estimación la función logística, y que además de obtener estimaciones de la probabilidad de un evento, identifica los factores de riesgo que determinan dichas probabilidades, así como la influencia relativa que éstos tienen sobre la variable dependiente.

El modelo se especifica de la siguiente manera:

$\operatorname{Pr}($ Hipogonadismo $=1 \mid x)=$ 1 $\overline{1+e^{-\left(\beta 0+\beta 1 \text { edad }+\beta 2 \text { edad }^{2}+\beta 3 \text { CD } 4+\beta 4 \text { LH }+\beta 5 \text { FSH }\right)}}$ 
En donde, CD4 representa la cantidad de linfocitos presentes en el sistema inmune de los pacientes, LH es una de las hormonas que influye en los niveles de testosterona, FSH se define como la hormona que regula los procesos de crecimiento y procesos reproductivos en los hombres, la edad representa los años de vida de los pacientes y edad ${ }^{2}$, dicha variable elevada al cuadrado.

\section{RESULTADOS}

La muestra incluye 114 hombres infectados de $\mathrm{VIH}$, con una edad media de 35 años, de los cuales, el 6\% padecen de hipogonadismo. Además, presentan -en promedio- niveles de testosterona de $274.45 \mathrm{ng} / \mathrm{dl}$. Así mismo, la media de recuento de células CD4 es de 376.04 $\mathrm{c} / \mathrm{mm}^{3}$, mientras que la media de LH y FSH son de $5.89 \mathrm{mlU} / \mathrm{ml}$ y $5.68 \mathrm{mlU} / \mathrm{ml}$, respectivamente.

Para estimar el modelo propuesto se empleó el método de máxima verosimilitud con un nivel de confianza del 95\% y utilizando errores estándares robustos. La Tabla 1 muestra los resultados:

Tabla 1. Modelo Logit estimado sobre la incidencia de hipogonadismo

\begin{tabular}{|c|c|c|c|}
\hline \multicolumn{2}{|c|}{ Variables } & \multirow{2}{*}{$\begin{array}{l}\text { Coeficiente } \\
0,1755448\end{array}$} & \multirow{2}{*}{$\begin{array}{c}\text { Error estándar } \\
\text { 0,1987728 }\end{array}$} \\
\hline Edad & & & \\
\hline Edad $^{2}$ & & $-0,0016873$ & 0,0023623 \\
\hline \multicolumn{4}{|l|}{ CD4 } \\
\hline & $201-350$ & 0,4365459 & 0,5871012 \\
\hline & $351-500$ & 0,024036 & 0,577898 \\
\hline & $501-800$ & 0,5951623 & 0,5887331 \\
\hline LH & & $-0,2714806$ & 0,1965562 \\
\hline FSH & & 0,0466176 & 0,1438539 \\
\hline Constante & & -4.216784 & 1,811865 \\
\hline
\end{tabular}

FSH: hormona folículo estimulante, LH: hormona luteinizante.

El modelo estimado reporta coeficientes con respecto al logaritmo de la razón de probabilidad, los cuales no son interpretables directamente. Por ende, se procedió a estimar los efectos marginales que asemejan la interpretación de un modelo de regresión lineal. Los efectos de las variables continuas del modelo fueron evaluados por el incremento de una desviación estándar, mientras que para la variable CD4, la categoría 1 sirve como base para la interpretación de las demás categorías (Tabla 2).

Tabla 2. Efectos marginales

\begin{tabular}{|c|c|c|}
\hline Variables & & Efecto \\
\hline \multicolumn{3}{|l|}{ Edad } \\
\hline & SD & 0,074 \\
\hline \multicolumn{3}{|l|}{ Edad $^{2}$} \\
\hline & SD & $-0,106$ \\
\hline \multicolumn{3}{|l|}{ CD4 } \\
\hline 2 vs 1 & & 0,103 \\
\hline 3 vs 1 & & 0,006 \\
\hline 4 vs 1 & & 0,138 \\
\hline 3 vs 2 & & $-0,097$ \\
\hline 4 vs 2 & & 0,035 \\
\hline 4 vs 3 & & 0,132 \\
\hline \multicolumn{3}{|l|}{$\mathrm{LH}$} \\
\hline & SD & $-0,084$ \\
\hline \multicolumn{3}{|l|}{$\mathrm{FSH}$} \\
\hline & SD & 0,038 \\
\hline
\end{tabular}

FSH: hormona folículo estimulante, LH: hormona luteinizante, SD: desviación estándar. 
Por lo tanto, un aumento de una desviación estándar, que corresponde a 12 años en la edad de los participantes, incrementa la probabilidad de contraer hipogonadismo en un 7.4\%. Luego, al observar las probabilidades de contraer la afección según los niveles de CD4 propuesto, se evidenció que para un participante con niveles de CD4 correspondientes a la categoría 2, la probabilidad de contraer hipogonadismo se incrementa en un $10 \%$ y para aquel en la categoría 3 , aumenta en un $0.06 \%$. Se puede notar que entre estos dos resultados existe una reducción en la probabilidad de padecer hipogonadismo, por lo tanto, obedece la hipótesis propuesta, esto es, a mayores niveles de CD4 existentes, menores posibilidades de sufrir de esta afección; es decir, el sujeto en la categoría 2 es más propenso a adquirir hipogonadismo que el de la categoría 3 con respecto a la categoría 1. La probabilidad para el participante de la categoría 4 se incrementa en un $13 \%$, este resultado se desliga de la hipótesis, por esto se puede inducir a priori que no hay una relación entre un aumento de las células CD4 con la presencia - ausencia de hipogonadismo. El análisis realizado se aplicó a cada par de categorías y con distintas bases, manteniéndose el patrón expuesto. Por otra parte, un incremento de una desviación estándar en los valores de LH reduce la probabilidad de padecer de hipogonadismo en un $8.4 \%$, resultado nada sorpresivo dado que la testosterona es sintetizada principalmente por la acción de la LH. En cuanto a la FSH, un incremento de una desviación estándar incrementa la probabilidad de ser diagnosticado con hipogonadismo en 3.8\%.

Adicionalmente, se calcularon las probabilidades medias de ser diagnosticado con hipogonadismo de acuerdo a los rangos de CD4 propuestos, manteniendo todas las demás variables constantes (en la media); los resultados se muestran en la Figura 1.

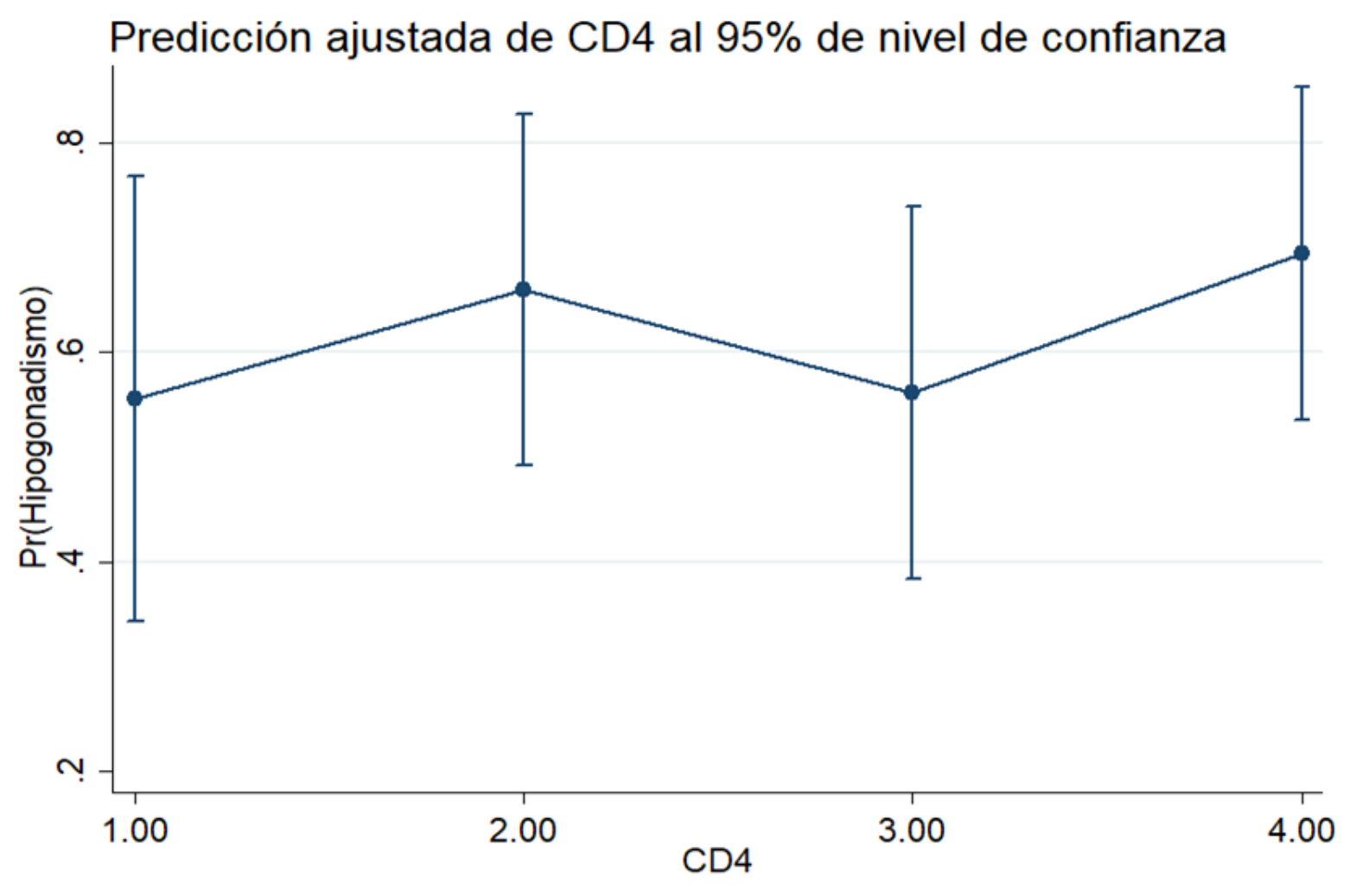

Figura 1. Probabilidad de ser diagnosticado con hipogonadismo de acuerdo a los rangos de CD4. 
Los coeficientes muestran la probabilidad de padecer la afección según los niveles de CD4 en los pacientes. Por lo tanto, la probabilidad de padecer hipogonadismo para el paciente promedio, con niveles de CD4 en un rango de 0 a $200 \mathrm{c} / \mathrm{mm}^{3}$, es del 15\%; para el paciente promedio con niveles de entre 201 a $350 \mathrm{c} / \mathrm{mm}^{3}$ es del $6 \%$, para quienes tienen entre 351 y $500 \mathrm{c} / \mathrm{mm}^{3}$ es del $56 \%$, y aquellos con niveles entre 500 y $800 \mathrm{c} / \mathrm{mm}^{3}$ es del $69 \%$.

De igual manera, se estimó la probabilidad del paciente promedio de tener hipogonadismo a partir de incrementos constantes de 10 años en la edad desde el valor mínimo de la variable, los resultados se encuentran en la Figura 2. Las estimaciones revelan incrementos considerables en la probabilidad de padecer de hipogonadismo a medida que la edad del paciente promedio se incrementa 10 años.

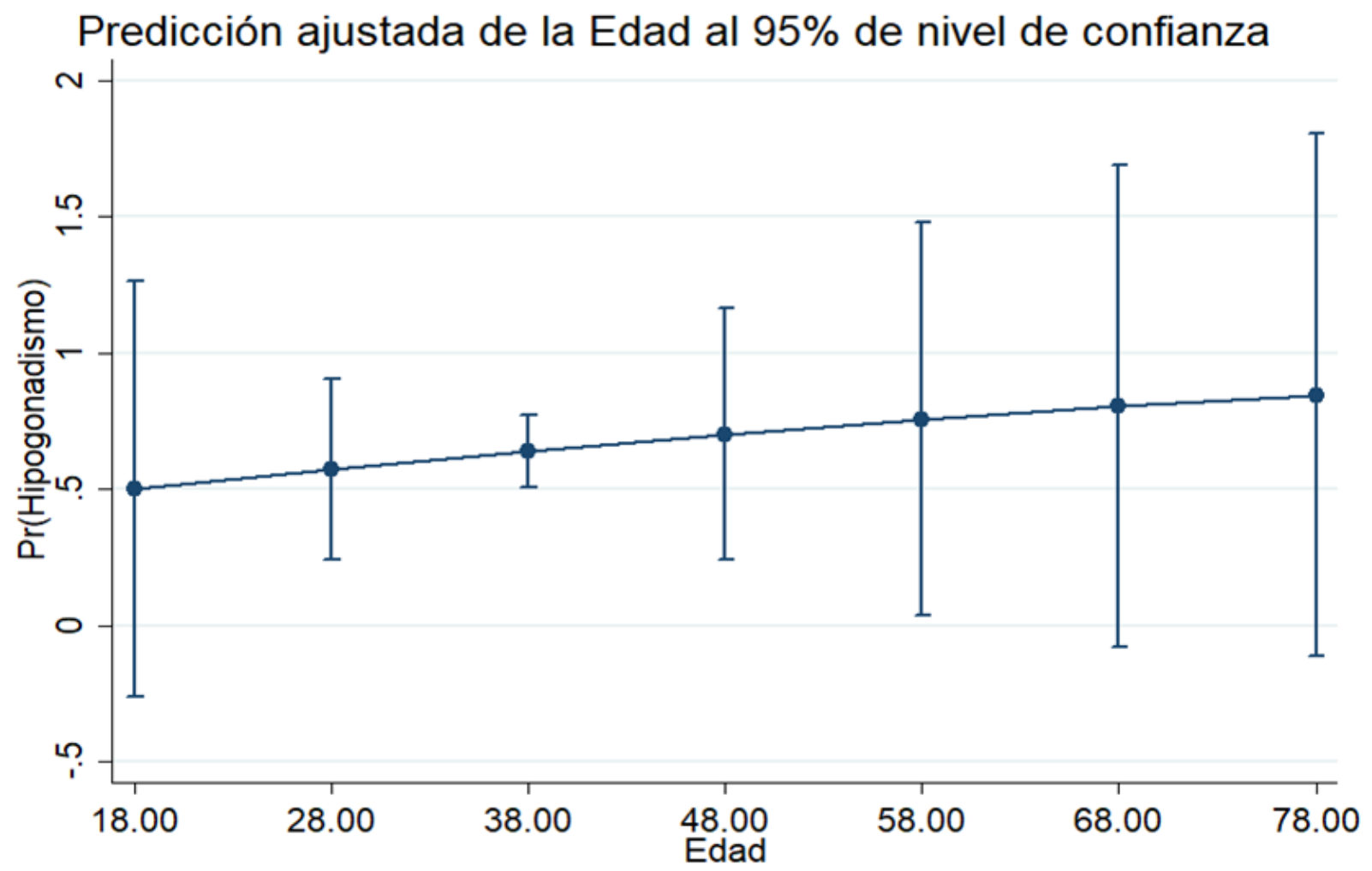

Figura 2. Probabilidad de desarrollar hipogonadismo según la edad.

Finalmente, para determinar la significancia estadística de los factores de riesgo asociados a la posibilidad de ser o no diagnosticado con hipogonadismo, se calculó el valor z y su valor p asociado para cada variable independiente (Tabla 3).

Tabla 3. Prueba estadística de significancia individual de las variables

\begin{tabular}{|c|c|c|c|}
\hline Variables & Coeficiente & $\mathbf{z}$ & $P>Z$ \\
\hline Edad & 0,1755448 & 0,88 & 0,377 \\
\hline Edad $^{2}$ & $-0,0016873$ & $-0,71$ & 0.475 \\
\hline \multicolumn{4}{|l|}{ CD4 } \\
\hline $201-350$ & 0,4365459 & 0,74 & 0,457 \\
\hline $351-500$ & 0,024036 & 0,04 & 0,967 \\
\hline $501-800$ & 0,5951623 & 1,01 & 0,312 \\
\hline $\mathrm{LH}$ & $-0,2714806$ & -1.38 & 0,167 \\
\hline $\mathrm{FSH}$ & 0,0466176 & 0,32 & 0,746 \\
\hline Constante & -4.216784 & $-0,99$ & 0,325 \\
\hline
\end{tabular}

FSH: hormona folículo estimulante, LH: hormona luteinizante. 
Como se puede observar en la tabla anterior, todos los valores $\mathrm{p}$ calculados asociados a cada variable independiente se encuentran por encima del $5 \%$ del nivel de significancia. Por lo tanto, no existe evidencia estadística que permita corroborar el impacto de los niveles de CD4, FSH, LH y la edad sobre la presencia de hipogonadismo.

Durante la era pre-Tratamiento Antirretroviral de Gran Actividad (TARGA), varios análisis exponen los desórdenes hormonales presentes en los pacientes con VIH (13-16). Dobs et al. (17) por ejemplo, realizaron pruebas en 70 hombres seropositivos de los cuales 19 eran asintomáticos, 9 portaban ARC (complejo relacionado con el SIDA) y 42 tenían SIDA. Los resultados reflejaron que 20 de 40 hombres con SIDA eran hipogonadales, las concentraciones de testosterona en ambos: ARC (292 $\pm 70 \mathrm{ng} / \mathrm{dl})$ y SIDA (401 $\pm 30 \mathrm{ng} / \mathrm{dl}$ ) eran significativamente menores que los hombres asintomáticos (567 \pm $49 \mathrm{ng} / \mathrm{dl}$ ) o los hombres normales (606 $\pm 121 \mathrm{ng} /$ dl). Fue así como asociaron el hipogonadismo con la disminución de los linfocitos y la pérdida de peso.

Adiferencia de Dobs, Croxsony col.(18) realizaron estas pruebas a 85 hombres homosexuales, donde el $32 \%$ de pacientes y el $50 \%$ de aquellos en la etapa del SIDA sufrían hipogonadismo primario; concluyendo que el mecanismo de la afección no estaba claramente definido, ya que el hipogonadismo iba acompañado de altas concentraciones de LH seguido de daños testiculares.

Años más tarde, Raffi et. al. (19) analizó las funciones endocrinas en 98 personas, 73 hombres y 25 mujeres. El $15 \%$ de los hombres evaluados, padecían hipogonadismo y el $29 \%$ de aquellos con SIDA tenían los menores niveles de testosterona. Esta carencia se relacionó con la deficiencia funcional del eje hipotalámicohipofisario, correlacionado con el grado de enfermedad, es decir, la pérdida de peso y el bajo recuento de células CD4.

A esta presunción se sumó Laudat y otros (11), quienes tomando muestras de globulina fijadora de hormonas sexuales, androstenediona, testosterona, $\mathrm{FSH}, \mathrm{LH}$, dihidrotestosterona, dehidroepiandrosterona y cortisol en 58 hombres homosexuales portadores del $\mathrm{VIH}$, notaron que los niveles de estas hormonas se vinculaban con los recuentos de células CD4, mostrando que el hipogonadismo sucedía a medida que las células CD4 disminuían.
En el mismo año, Wagner et. al. (20) al examinar a 234 hombres diagnosticados con $\mathrm{VIH}$, por medio de pruebas de sangre para medir la cantidad de testosterona y marcadores de inmunodeficiencia, evidenciaron que la cantidad de testosterona se asociaba con un bajo recuento de células CD4 (en las últimas etapas de la enfermedad), uso de megestrol y la edad. El análisis de las variables reflejó que solo la edad y el uso de medicamentos como megestrol, eran el origen de baja testosterona (20).

Grinspoon et. al (16) por su parte, estudió a 77 hombres con $\mathrm{VIH}$, de los cuales, el $49 \%$ sufrían de hipogonadismo, diagnosticados a través de la medición de los niveles de testosterona (testosterona $<12 \mathrm{pg} / \mathrm{mL}$ ) y con un modelo de regresión lineal, mostraron una mayor diferencia en los recuentos de CD4 y el peso entre los pacientes con mayor y menor grado de testosterona, alegando que toda la testosterona se ligaba a la cantidad de masa corporal magra y muscular.

Rietschel et. al. (8) examinaron los niveles de testosterona en 90 hombres portadores de $\mathrm{VIH}$, con menos del $90 \%$ de su peso ideal o con pérdidas superiores del $10 \%$ de su peso antes de la enfermedad; notaron que el $71 \%$ de los participantes estaba recibiendo el tratamiento antirretroviral. Ninguna correlación fue hallada entre el peso, recuento de CD4, y otros factores clínicos. Adicionalmente, a través del test de Dunnett, demostraron que el $20 \%$ de los contagiados de VIH eran hipogonadales. Además de que las similares tasas de incidencia de hipogonadismo en los pacientes recibiendo TARGA (21\%) y los que no (15\%), constatan la irrelevancia del tratamiento en los niveles de testosterona.

Crum et. al. (21) en su trabajo seminal, evidenciaron que la baja cantidad de CD4 no era causa del hipogonadismo, asociando la enfermedad con el uso de drogas ilícitas, esteroides y alcohol, además no observaron influencia del TARGA en su muestra de contagiados. En esta línea, múltiples estudios desligaron el uso de antirretrovirales y la cantidad de CD4 como causa del hipogonadismo (7,22-24).

Contrario a los hallazgos de estudios anteriores, Bajaj et. al. (12) tomando una muestra de 81 hombres con VIH de los cuales, el 25.9\% padecían de hipogonadismo, y realizando recuento de CD4, medición de testosterona, LH, FSH e índice de masa corporal, mostraron que la afección era 
común entre los casos con una disminución en sus células CD4. Este último trabajo se desliga de la línea de tiempo respecto a las conclusiones provistas en estudios precedentes y vuelca el interés hacia las conjeturas iniciales sobre los determinantes de hipogonadismo en pacientes con $\mathrm{VIH}$, debido a que en la era pre tratamiento, la afección fue relacionada con la disminución de los niveles de CD4, y en la era post tratamiento, con el uso de medicamentos contra el $\mathrm{VIH}$ e incluso con factores independientes del virus. Evidentemente, la incidencia de la afección en los portadores de VIH continua siendo un generador de hipótesis sobre la etiología del hipogonadismo, lo que alienta a la comunidad científica a proveer nuevas explicaciones del fenómeno en estudio.

\section{DISCUSIÓN}

El análisis inferencial realizado evidenció la ausencia de un impacto estadísticamente significativo de los niveles de células CD4 sobre la posibilidad de contraer hipogonadismo en los hombres con VIH. Es decir, la etapa del VIH en la que se encuentre el paciente es independiente de padecer hipogonadismo; los hallazgos en este estudio coinciden con los resultados de trabajos $(4,7,8,25)$.

Además, al evaluar si la edad de los participantes tiene algún efecto sobre la presencia de la enfermedad, se halló que este factor tampoco tiene relevancia en la posibilidad de afección de esta, a diferencia del trabajo de Bajaj et al. (12) quien si asocia la edad con el hipogonadismo en los sujetos con el virus. Se analizó también LH y FSH, hormonas relacionadas con la producción de testosterona, pero no se encontró algún patrón significativo de estas sobre el hipogonadismo en los participantes.

Todas las variables analizadas, fueron interpretadas a través del agente promedio de muestra, de esta forma, fueron valoradas los resultados según las limitaciones correspondientes. Para siguientes estudios se recomienda realizar un seguimiento en el desarrollo del virus y niveles de testosterona de cada paciente, es decir, se sugiere efectuar el mismo análisis inferencial, pero utilizando datos de los pacientes a través del tiempo, de tal forma, que se infiera los efectos de las variables analizadas a través de la información de cada paciente en distintas etapas de la enfermedad. Esto implica utilizar métodos estadísticos aplicados a datos de panel, una mezcla de datos de corte transversal con series de tiempos.
Adicionalmente se sugiere incluir variables sociodemográficas como variables de control, que regule las estimaciones.

\section{REFERENCIAS BIBLIOGRÁFICAS}

1. Organización de las Naciones Unidas. Estadísticas Mundiales sobre el VIH [Internet]. ONUSIDA. 2018 [citado 2020 Feb 10]. Disponible en: http:// www.unaids.org/sites/default/files/media_asset/ UNAIDS_FactSheet_es.pdf

2. Wong N, Levy M, Stephenson I. Hypogonadism in the HIV-Infected Man. Curr Treat options Infect Dis. 2017;9(1):104-16.

3. 3. Organización Mundial de la Salud. Estrategia mundial del sector de la Salud contra el VIH 20162021. Ginebra; 2016.

4. Crum NF, Furtek $\mathrm{KJ}$, Olson $\mathrm{PE}$, Amling $\mathrm{CL}$, Wallace MR. A review of hypogonadism and erectile dysfunction among HIV-infected men during the pre- and post-HAART eras: diagnosis, pathogenesis, and management. AIDS Patient Care STDS. 2005 Oct;19(10):655-71.

5. Broder M. HIV and Male Hypogonadism: A Persistent Challenge Even With Effective ART. 2017.

6. Sunchatawirul K, Tantiwongse K, Chathaisong P, Thongyen S, Chumpathat N, Manosuthi W. Hypogonadism among HIV-infected men in Thailand. Int J STD AIDS. 2012;23(12):876-81.

7. Ranabir S, Nungsangla P, Premita M, Shaini L, Biplab Singh N, Bhagyabati Devi S, Robinson N. Hypogonadism among HIV infected males and its correlation with CD4 count. J Evid Based Med Healthc. 2018 Aug 20;5:2507-11.

8. Rietschel P, Corcoran C, Stanley T, Basgoz N, Klibanski A, Grinspoon S. Prevalence of hypogonadism among men with weight loss related to human immunodeficiency virus infection who were receiving highly active antiretroviral therapy. Clin Infect Dis. 2000 Nov;31(5):1240-4.

9. Bhasin S, Cunningham GR, Hayes FJ, Matsumoto AM, Snyder PJ, Swerdloff RS, et al. Testosterone therapy in men with androgen deficiency syndromes: an Endocrine Society clinical practice guideline. J Clin Endocrinol Metab. 2010 Jun;95(6):2536-59.

10. Noda Albelo AL, Vidal Tallet LA, Pérez Lastre JE, Cañete Villafranca R. Interpretación clínica del conteo de linfocitos T CD4 positivos en la infección por VIH. Revista cubana med. 2013;52(2):118-27.

11. Laudat A, Blum L, Guéchot J, Picard O, Cabane J, Imbert JC, et al. Changes in systemic gonadal and adrenal steroids in asymptomatic human immunodeficiency virus-infected men: relationship with the CD4 cell counts. Eur J Endocrinol. 1995 Oct;133(4):418-24.

12. Bajaj S, Pathak Y, Varma S, Verma S. Metabolic Status and Hypogonadism in Human Immunodeficiency Virus-infected Males. Indian J Endocrinol Metab. 2017;21(5):684-7. 
13. Dobs AS, Few WL 3rd, Blackman MR, Harman SM, Hoover DR, Graham NM. Serum hormones in men with human immunodeficiency virusassociated wasting. J Clin Endocrinol Metab. 1996 Nov;81(11):4108-12.

14. Merenich JA, McDermott MT, Asp AA, Harrison SM, Kidd GS. Evidence of endocrine involvement early in the course of human immunodeficiency virus infection. J Clin Endocrinol Metab. 1990 Mar;70(3):566-71.

15. Christeff N, Gharakhanian S, Thobie N, Rozenbaum W, Nunez EA. Evidence for changes in adrenal and testicular steroids during HIV infection. J Acquir Immune Defic Syndr. 1992;5(8):841-846.

16. Grinspoon SK, Bilezikian JP. HIV Disease and the Endocrine System. N Engl J Med. 1992 Nov 5;327(19):1360-5.

17. Dobs AS, Dempsey MA, Ladenson PW, Polk BF. Endocrine disorders in men infected with human immunodeficiency virus. Am J Med. 1988 Mar;84(3 Pt 2):611-6.

18. 18. Croxson TS, Chapman WE, Miller LK, Levit CD, Senie R, Zumoff B. Changes in the hypothalamicpituitary-gonadalaxis in human immunodeficiency virus-infected homosexual men. J Clin Endocrinol Metab. 1989 Feb;68(2):317-21.

19. Raffi F, Brisseau JM, Planchon B, Rémi JP, Barrier $\mathrm{JH}$, Grolleau JY. Endocrine function in 98 HIVinfected patients: a prospective study. AIDS. 1991 Jun;5(6):729-33.
20. Wagner G, Rabkin JG, Rabkin R. Illness stage, concurrent medications, and other correlates of low testosterone in men with HIV illness. J Acquir immune Defic Syndr Hum retrovirology Off Publ Int Retrovirology Assoc. 1995 Feb;8(2):204-7.

21. Crum NF, Riffenburgh RH, Wegner S, Agan BK, Tasker SA, Spooner KM, et al. Comparisons of causes of death and mortality rates among HIVinfected persons: analysis of the pre-, early, and late HAART (highly active antiretroviral therapy) eras. J Acquir Immune Defic Syndr. 2006 Feb;41(2):194-200.

22. Rochira V, Zirilli L, Orlando G, Santi D, Brigante G, Diazzi $C$, et al. Premature decline of serum total testosterone in HIV-infected men in the HAARTera. PLoS One. 2011;6(12):e28512.

23. Crum-Cianflone NF, Bavaro M, Hale B, Amling C, Truett A, Brandt $C$, et al. Erectile dysfunction and hypogonadism among men with HIV. AIDS Patient Care STDS. 2007 Jan;21(1):9-19.

24. Guaraldi G, Luzi K, Murri R, Granata A, De Paola $M$, Orlando $G$, et al. Sexual dysfunction in HIVinfected men: role of antiretroviral therapy, hypogonadism and lipodystrophy. Antivir Ther. 2007;12(7):1059-65.

25. Klein RS, Lo Y, Santoro N, Dobs AS. Androgen levels in older men who have or who are at risk of acquiring HIV infection. Clin Infect Dis an Off Publ Infect Dis Soc Am. 2005 Dec;41(12):1794-803.

\section{ACERCA DE LOS AUTORES}

1. Fernando Rafael Rodríguez Ibarra. Especialista en Medicina Interna, Clínica Guayaquil, Ecuador. Médico tratante de Medicina Interna, Hospital General de Quevedo - IESS, Quevedo, Ecuador. Clínica Guayaquil, Guayaquil-Ecuador.

ORCID: 0000-0001-6436-8021

2. Ixsoon Alberto Castillo. Coordinador de la Unidad de Atención Integral de VIH, Hospital de Especialidades Eugenio Espejo, Ecuador.

3. Patricia Lisseth Borrero Párraga. Médico posgradista de Medicina Interna, Universidad de Especialidades Espíritu Santo, Clínica Guayaquil, Guayaquil-Ecuador.

ORCID: 0000-0003-0280-4339

4. Tatiana Carolina Marcillo Pin. Médico posgradista de Medicina Interna, Universidad de Especialidades Espíritu Santo, Clínica Guayaquil, Guayaquil-Ecuador.

ORCID: 0000-0001-6658-5505 